\title{
Hypoxia-inducible expression of the mouse carbonic anhydrase IX demonstrated by new monoclonal antibodies
}

\author{
MARTINA TAKACOVA ${ }^{1}$, MONIKA BARATHOVA $^{1}$, ALZBETA HULIKOVA $^{1}$, \\ ANNA OHRADANOVA ${ }^{1}$, JURAJ KOPACEK ${ }^{1}$, SEPPO PARKKILA ${ }^{2}$, \\ JAROMIR PASTOREK $^{1}$, SILVIA PASTOREKOVA ${ }^{1}$ and MIRIAM ZATOVICOVA ${ }^{1}$ \\ ${ }^{1}$ Institute of Virology, Centre of Molecular Medicine, Slovak Academy of Sciences, Dubravska cesta 9, \\ 84505 Bratislava, Slovak Republic; ${ }^{2}$ Institute of Medical Technology, University of Tampere \\ and Tampere University Hospital, Biokatu 6, 33520 Tampere, Finland
}

Received June 13, 2007; Accepted July 26, 2007

\begin{abstract}
CA IX is a transmembrane carbonic anhydrase isoenzyme predominantly expressed in human tumors in response to hypoxia and functionally implicated in adaptation of tumor cells to hypoxic stress via control of $\mathrm{pH}$ and cell adhesion. Intense investigations of the human CA IX as a hypoxic marker and a therapeutic target have been facilitated by specific monoclonal antibodies. However, no such reagents existed for the mouse CA IX ortholog. We generated five new anti-mouse CA IX monoclonal antibodies AM1-4, AM4-3, AM27-4, AM34-7 and AM35-1 produced using CA IX-deficient mice. The antibodies are suitable for various immunodetection methods including immunoblotting and immunohistochemistry. Using these reagents we show that the mouse CA IX is expressed in three out of nine tested mouse cell lines, namely in L929, MEF and TSA and is regulated by hypoxia and cell density similarly to human CA IX. We also demonstrate that the mouse CA IX exhibits hypoxia-related expression pattern in multicellular spheroids and in tumor xenografts. Our results indicate the use of the mouse model as suitable for further studies of CA IX role in tumor development and for its pre-clinical investigations. The new monoclonal antibodies represent potent tools for accomplishment of these future studies.
\end{abstract}

Correspondence to: Dr Miriam Zatovicova, Institute of Virology, Centre of Molecular Medicine, Slovak Academy of Sciences, Dubravska cesta 9, 84505 Bratislava, Slovak Republic

E-mail: viruzato@savba.sk

Abbreviations: CA, carbonic anhydrase; IHC, immunohistochemistry; MAb, monoclonal antibody; PG, proteoglycan; GST, glutathione-S transferase

Key words: carbonic anhydrase IX, mouse, monoclonal antibodies, hypoxia, CA IX deficient mice

\section{Introduction}

Carbonic anhydrases (CAs) are zinc-binding enzymes that catalyze the reversible conversion between carbon dioxide and carbonic acid, in a reaction that involves facilitated hydration of $\mathrm{CO}_{2}$ to $\mathrm{H}_{2} \mathrm{CO}_{3}$ followed by the spontaneous dissociation of $\mathrm{H}_{2} \mathrm{CO}_{3}$ into bicarbonate and a proton (1). Via this catalytic activity, CAs significantly contribute to modulation of ion transport and maintenance of acid-base balance in cells and tissues of virtually all living organisms. In mammals, there are sixteen $\mathrm{CA}$ isoforms that show considerable diversity in their molecular properties, subcellular localization, distribution in tissues, expression level, enzyme activity and kinetic properties as well as in their affinity and sensitivity to inhibitors. Thirteen active isoenzymes (CA I-IV, VA, VB, VI, VII, IX, XII-XV) possess a conserved active site that contains three histidine residues necessary for the coordination of a zinc ion and the fourth histidine residue functioning as a proton shuttle. These catalytically active CAs are mostly present in differentiated cells of the normal tissues, where they play specific roles in different physiological and pathological situations $(2,3)$.

CA IX isoform has been cloned from human cancer cells and differs from the other CA isoenzymes by its predominant association with various human tumors, including carcinomas of the cervix uteri, kidney, lung, colon, breast, brain, ovary and other types of neoplasia $(4,5)$. In the normal tissues, significant CA IX expression is restricted to gastrointestinal tract, namely to epithelia of the stomach, small intestine and gallbladder $(6,7)$. This tumor-related expression pattern of CA IX is primarily caused by the strong transcriptional activation of the human CA9 gene by hypoxia via the hypoxiainducible transcription factor (HIF). In weakly oxygenated cells, HIF assembles from the oxygen-sensitive $\alpha$ subunit and constitutive $\beta$ subunit, binds to hypoxia response element present in the CA9 promoter near the transcription initiation site and induces the CA9 gene expression (8). In the normoxic conditions, transcription of the human CA9 gene correlates with high cell density connected with pericellular hypoxia (9). Functional studies of the human CA IX protein indicate 
its involvement in modulation of $\mathrm{E}$ cadherin-mediated cell adhesion via direct interaction with $\beta$ catenin (10). CA IX also actively participates in $\mathrm{pH}$ regulation across the plasma membrane and contributes to acidification of extracellular microenvironment in response to hypoxia (11).

Due to the tight hypoxic regulation and functional implication in tumor biology, human CA IX protein has been extensively studied with respect to its distribution in different tumor types, relationship to intratumoral hypoxia, to prognostic parameters and to its usefulness as a therapeutic target. Based on these studies, CA IX has been proposed to serve as a surrogate marker of tumor hypoxia with the predictive and/or prognostic values and as a molecular target for immunotherapy of renal carcinomas $(5,12)$. Moreover, RNA interference experiments to block the hypoxia-induced expression of CA IX led to growth delay and reduced clonogenic survival of hypoxic cancer cells, suggesting that CA IX represents a promising functional target for anticancer therapy (13).

Investigations of the human CA IX protein (hCA IX) have been facilitated by the availability of several highly specific monoclonal antibodies, including the M75 MAb against the N-terminal proteoglycan (PG) domain of CA IX and a series of the recently described MAbs directed to its catalytic CA domain $(14,15)$. However, except M75 that can bind to the rat CA IX protein, none of these antibodies recognizes the mouse CA IX ortholog (mCA IX) and thus all previous studies of CA IX in the mouse model used rabbit polyclonal serum (16-19). Therefore, we decided to generate monoclonal antibodies that would represent specific tools for detection of the mouse CA IX protein in mouse cell lines and tissues as well as for its functional investigations and targeting in pre-clinical studies using the mouse model. Here we describe and characterize five new monoclonal antibodies that are suitable for such purposes. Using these monoclonal antibodies we demonstrate for the first time that the mouse CA IX protein is induced by hypoxia as well as by increased cell density in culture and displays similar intratumoral distribution as the human CA IX protein.

\section{Materials and methods}

Production of hybridomas. CA IX-deficient mice $(16,20)$ were used for immunization at the age of 8-10 weeks. The mice were injected intraperitoneally (i.p.) with $100 \mu \mathrm{g}$ of purified mouse CA IX protein fused to GST (16) and bound to glutathion-S sepharose in $0.5 \mathrm{ml}$ PBS. This first injection was used in combination with Titer Gold Max adjuvant (Sigma, St. Louis, MA). After three weeks, the mice were boosted i.p. without the adjuvant, splenocytes were harvested 3 days later and fused with the Sp2/0 myeloma cells as described previously (15). Hybridomas were selected in DMEM-HAT medium containing hypoxanthine, aminopterin and thymidine, and screened for the specific reactivity towards CA IX by ELISA as described below. Positive hybridomas were subcloned by limiting dilution, expanded, subjected to freezing-refreezing and repeatedly tested for the reactivity to CA IX. The protocol for the monoclonal antibody production was approved by the Slovak Veterinary Administration, in accordance with EU regulations.
Cell culture. Hybridoma cell lines were grown in DMEM medium supplemented with 10\% FCS (BioWhittaker, Verviers, Belgium), $2 \mathrm{mM}$ glutamine and $40 \mu \mathrm{g} / \mathrm{ml}$ gentamicin (Lek Slovenia) at $37^{\circ} \mathrm{C}$ in air with $5 \% \mathrm{CO}_{2}$. The same cultivation conditions were applied to cell lines that were used either as a source of CA IX antigen or as negative controls, including the mouse NIH-mCA IX transfected cells, corresponding mock-transfected NIH3T3 controls (NIH-neo), mouse L929 fibroblasts, TSA mouse mammary carcinoma cells, mouse embryonic fibroblasts (MEF-wt) and MEF deficient in HIF- $1 \alpha$ (MEF-KO, kindly provided by Dr R.S. Johnson, University of California, San Diego), mouse hepatoma cells Hepac4 deficient in HIF-1ß, mouse hepatoma cells Hepa1c1c7 (from Professor L. Poellinger, Karolinska Institute, Stockholm), mouse 15P-1 line with the characteristics of Sertoli cells, mouse testicular GC1-spg cells, canine kidney MDCK cells transfected with the mouse Car9 cDNA (MDCK-mCA IX), mock-transfected MDCK-neo cells, human HT29 colon carcinoma cells, virus-induced rat sarcoma XC cells and Rat2TK- rat immortalized fibroblasts (from Dr J. Zavada, Institute of Molecular Genetics, Prague). Hypoxic treatments were performed in an anaerobic workstation (Ruskin Technologies, Bridgend, $\mathrm{UK}$ ) in $2 \% \mathrm{O}_{2}, 5 \% \mathrm{CO}_{2}, 10 \% \mathrm{H}_{2}$ and $83 \%$ $\mathrm{N}_{2}$ at $37^{\circ} \mathrm{C}$.

MEF-wt spheroids were pre-formed from 600 cells per $20 \mu 1$ of culture medium in drops hanging on the lid of tissue culture dish for three days at $37^{\circ} \mathrm{C}$. The resulting cell aggregates were transferred to Petri dish with a non-adherent surface and cultivated in suspension for an additional 11 days with the medium exchange every third day. The spheroids were then quickly fixed in Carnoy's fluid to preserve their integrity and embedded in paraffin.

Tumor xenografts were obtained by subcutaneous injection of $5 \times 10^{5}$ TSA cells in $200 \mu 1$ of phosphate-buffered saline (PBS) of 5-6 weeks old NuNu mice (Charles River, Germany). The tumors were allowed to grow for 25 days, then the animals were sacrificed by cervical dislocation, and the tumor as well as the stomach tissues were dissected and processed for immunohistochemistry as described below. The protocol for the animal handling in this study was approved by the Slovak Veterinary Administration, in accordance with EU regulations.

Transfection. The NIH3T3 and MDCK cells, respectively, were plated in $35-\mathrm{mm}$ Petri dishes to reach $\sim 70 \%$ density the following day. Transfection was performed with $2 \mu \mathrm{g}$ of the pSG5C-Car9 plasmid containing the full-length mouse Car9 cDNA, together with $200 \mathrm{ng}$ of pSV2neo plasmid using a GenePorter II transfection kit from Gene Therapy Systems (San Diego, CA). The transfected cells were subjected to 2-week selection in medium with Geneticin G418 (Invitrogen, Carlsbad, CA) in a concentration of $500 \mu \mathrm{g} / \mathrm{ml}$. The resistant colonies were cloned, tested for expression of the mouse CA IX protein by ELISA and/or immunoblotting with the polyclonal serum against the mouse CA IX (16) and expanded for further study.

ELISA screening. Screening of the positive hybridomas was performed by a sandwich ELISA. Microplate wells were coated overnight at $37^{\circ} \mathrm{C}$ with RIPA extract (specified below) 
of NIH-mCA IX cells, diluted 1:10 in PBS, and in parallel with analogous extract of NIH-neo cells as a negative control and/or $10 \mathrm{ng} /$ well of GST-mCA IX, and GST as control. After blocking with $10 \%$ FCS in PBS, the coated wells were incubated with undiluted culture media from individual hybridomas. Peroxidase-labelled swine anti-mouse IgG (Sevapharma, Prague, Czech Republic) was used as detector.

Determination of isotypes. MAb isotypes were determined by ELISA using affinity purified rabbit anti-mouse IgG1, IgG2a, IgG2b, IgG3, IgM and IgA antibodies (Mouse Monoclonal Antibody Isotyping Reagents, Sigma) according to instructions of the manufacturer.

Immunofluorescence. Cells grown on glass coverslips were fixed in ice-cold methanol at $-20^{\circ} \mathrm{C}$ for $5 \mathrm{~min}$. Non-specific binding was blocked by incubation with PBS containing $1 \%$ BSA for $30 \mathrm{~min}$ at $37^{\circ} \mathrm{C}$. Then the cells were incubated with primary antibodies diluted in PBS with $0.5 \%$ BSA (PBSBSA) for $1 \mathrm{~h}$ at $37^{\circ} \mathrm{C}$, washed three times with PBS-BSA for $10 \mathrm{~min}$, incubated with anti-mouse FITC-conjugated horse antibody (Vector Laboratories, Burlingame, CA) diluted 1:300 in PBS-BSA for $1 \mathrm{~h}$ at $37^{\circ} \mathrm{C}$ and washed as before. Finally, the cells were mounted onto slides in the Fluorescent Mounting Media (Calbiochem, Darmstadt, Germany), analyzed with Leica DM4500B microscope and photographed with Leica DFC480 camera.

Immunoblotting. Cells grown in confluent monolayer were solubilized in ice-cold RIPA buffer (1\% Triton X-100, $1 \%$ deoxycholate, $0.15 \mathrm{M} \mathrm{NaCl}, \mathrm{pH}$ 7.2) containing Complete cocktail of protease inhibitors (Roche Diagnostics $\mathrm{GmbH}$, Mannheim, Germany) for $30 \mathrm{~min}$ on ice. The extracts were collected, cleared by centrifugation at $15,000 \mathrm{rpm}$ for $10 \mathrm{~min}$ at $4^{\circ} \mathrm{C}$ and stored at $-80^{\circ} \mathrm{C}$. Protein concentration in extracts was quantified by BCA protein assay reagent (Pierce, Rockford, IL). Total cellular extracts (50 $\mu \mathrm{g}$ of proteins/lane) were resolved in $10 \%$ SDS-PAGE gel. The proteins were then transferred onto PVDF membrane (Amersham Pharmacia Biotech, Buckinghamshire, UK). After blocking in 5\% non-fat dry milk with $0.2 \%$ Nonidet P40 in PBS, the membrane was probed with MAbs (undiluted hybridoma media), washed and treated with secondary anti-mouse HRP-conjugated swine antibody diluted 1:7500 (Sevapharma, Prague, Czech Republic). Protein bands were visualized by enhanced chemiluminescence using ECL kit (Amersham Pharmacia Biotech).

Cell biotinylation and immunoprecipitation. Cells in monolayer of $70-80 \%$ confluence grown in a $10-\mathrm{cm}$ dish were washed with ice-cold buffer A (20 mM sodium hydrogen carbonate, $0.15 \mathrm{M} \mathrm{NaCl}, \mathrm{pH} 8.0$ ). Immediately before use, $1 \mathrm{mg}$ of NHS-LC-Biotin (Pierce) was dissolved in $50 \mu 1 \mathrm{DMSO}$, mixed with $4 \mathrm{ml}$ buffer $\mathrm{A}$, added to cells and incubated for $90 \mathrm{~min}$ at $4^{\circ} \mathrm{C}$. Finally, the cells were washed 5 times with buffer A and lysed as described above. Tested MAb in a volume of $1 \mathrm{ml}$ culture medium was bound to $25 \mu 150 \%$ suspension of Protein-G Sepharose (Pharmacia) for $2 \mathrm{~h}$ at RT. Biotinylated cell extract $(200 \mu 1)$ was pre-cleared with $20 \mu \mathrm{l}$ of $50 \%$ suspension of protein-G sepharose and then added to the bound MAb. Immunocomplexes collected on
protein-G sepharose were washed, boiled 5 min in Laemmli loading buffer and separated by SDS-PAGE gel (10\%) electrophoresis. Afterwards, the proteins were transferred onto PVDF membrane and revealed with peroxidase-conjugated streptavidin (1:1000, Pierce) followed by ECL.

Immunohistochemistry. Dissected tissues were fixed in $4 \%$ neutral buffered formalin or in Carnoy's fluid (absolute ethanolchloroform-glacial acetic acid in 6:3:1 ratio) and embedded in paraffin according to the standard histological procedures. Four- $\mu \mathrm{m}$ sections were placed on polysine-coated slides, dewaxed and rehydrated. The slides were first subjected to tissue pretreatment procedure at $125^{\circ} \mathrm{C}$ for $5 \mathrm{~min}$ in target retrieval solution (Pascal pressure chamber, DakoCytomation, Carpinteria, CA). The rest of the immunostaining procedure was performed in Dako Autostainer using the Dako Cytomation EnVision(r) ${ }^{+}$System-HRP (DAB) according to the manufacturer's instructions: a) peroxidase and protein block (10 min each); b) incubation for $1 \mathrm{~h}$ with primary antibody specific for the mouse CA IX (undiluted hybridoma medium) or PBS (negative control); c) incubation for $30 \mathrm{~min}$ with peroxidase-conjugated goat anti-mouse antibody diluted 1:1000 in antibody diluent (DakoCytomation). Staining was visualized with DAB solution for 1 min with 3,3'-diaminobenzidine as a chromogenic substrate. The slides were washed in PBS with $0.1 \%$ Tween-20 for 10 min after the step a, 2 times for 10 min after steps b and c, and three times in distilled water after visualisation with DAB. All incubations and washings were carried out at room temperature. Finally, the sections were counterstained with Mayer's hematoxylin, washed for $5 \mathrm{~min}$ and mounted in DePeX (Serva, Heidelberg, Germany). The stained sections were examined with Leica DM4500B microscope and photographed with Leica DFC480 camera.

\section{Results}

Generation and basic properties of the monoclonal antibodies to mouse CA IX. Production of specific mouse monoclonal antibodies against the mouse protein is not an easy task due to immune tolerance to self antigen. To overcome this problem, we have taken advantage of mice with the null mutation in Car9 gene, which do not express CA IX (16). We believed that these CA IX-deficient mice could recognize the mouse CA IX molecule as non-self and direct the humoral response to this protein. We made three independent efforts to obtain hybridomas of required specificity, in which the animals were immunized with three doses of cell extracts either from hypoxic L929 fibroblasts or from the mCA IX-transfected NIH3T3 cells, followed by an additional dose of the recombinant fusion GST-MCA IX protein. Although each fusion of splenocytes from the immunized animals resulted in generation of a high number of hybridomas $(1600,2400$ and 2400), no specific hybridomas were obtained. Finally, we succeeded with an immunization protocol containing two consecutive intraperitoneal doses of the recombinant fusion GST-mCA IX protein. The fusion brought 2,400 hybridomas, among which 44 produced monoclonal antibodies reactive in ELISA with the GST-mCA IX protein but not with the GST control. Subcloning and freezing-refreezing procedures led 
A
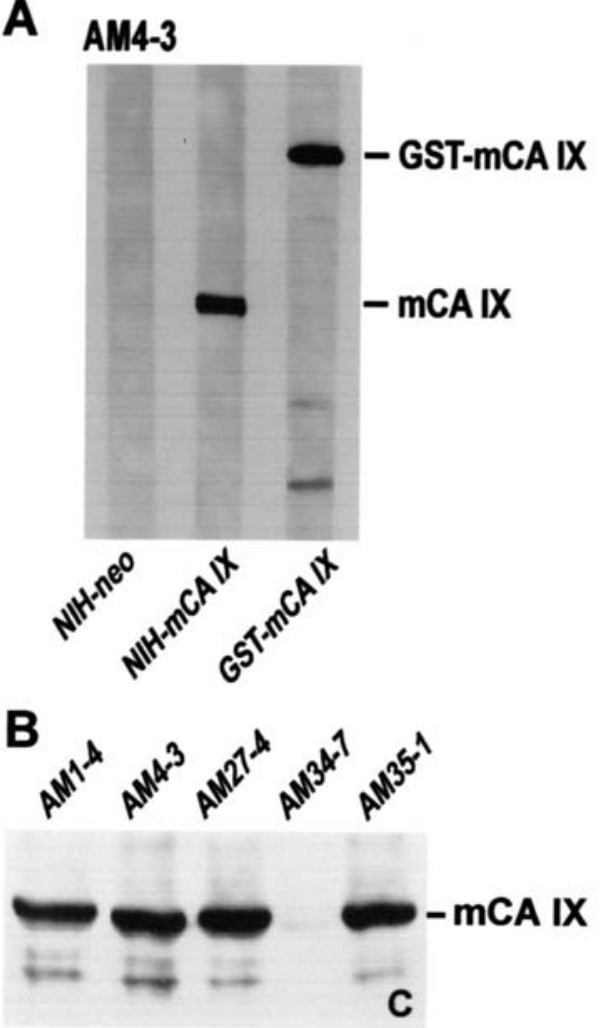

NIH-mCA IX

Figure 1. Specific reactivity of the new monoclonal antibodies against the mouse CA IX in immunoblotting and immunoprecipitation. (A) Immunoblot analysis of the new monoclonal antibodies for their specific reactivity against the mouse CA IX antigen expressed ectopically in the transfected NIH3T3 cells (NIH-mCA IX) compared to the mock-transfected control cells (NIH-neo), and against the mCA IX expressed in bacteria as a GST fusion protein (GST-mCA IX). The figure shows a representative blot obtained with the MAb AM4-3. (B) Four of five MAbs were capable of immunoprecipitating the mouse CA IX antigen from the extracts of the biotinylated $\mathrm{NIH}$-mCA IX transfectants.
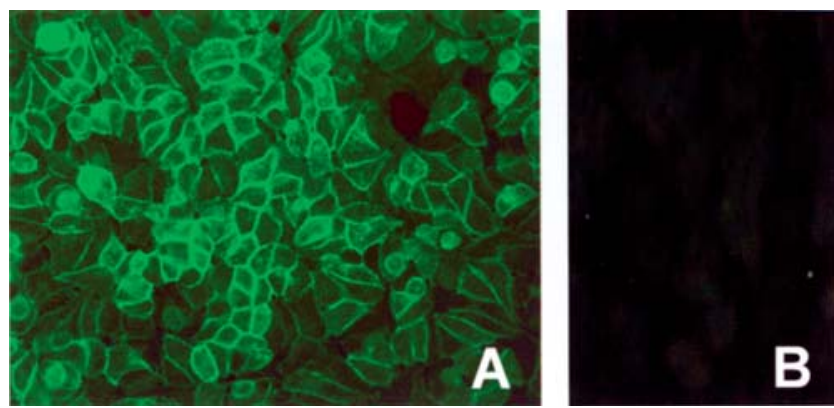

Figure 2. Immunofluorescence detection of mCA IX in MDCK cells transfected with the mouse Car9 cDNA. (A) The MDCK-mCA IX cells were grown to confluence, fixed by methanol and incubated with monoclonal antibody AM4-3 followed by FITC-conjugated anti-mouse antibodies. The antibody revealed the CA IX-specific plasma membrane staining. (B) Nontransfected MDCK-neo cells served as a negative control.

finally to five viable hybridoma cell lines producing antimouse CA IX monoclonal antibodies that were uniformly of IgG1 isotype. These results confirmed our anticipation that knock-out mice can be useful for the production of monoclonal

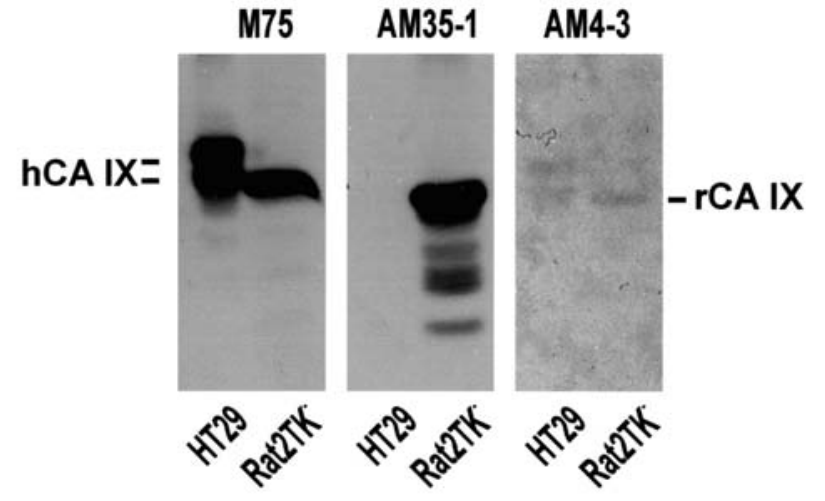

Figure 3. Immunoblotting analysis of the cross-reactivity of the new antimouse CA IX MAbs with the human and/or rat CA IX antigens. Out of the new MAbs, only AM35-1 showed cross-reactivity with the rat CA IX, but not with the human CA IX. M75 MAb was used as a positive control for detection of both human and rat antigens. AM4-3 as well as the other new MAbs did not show any cross-species reactivity.
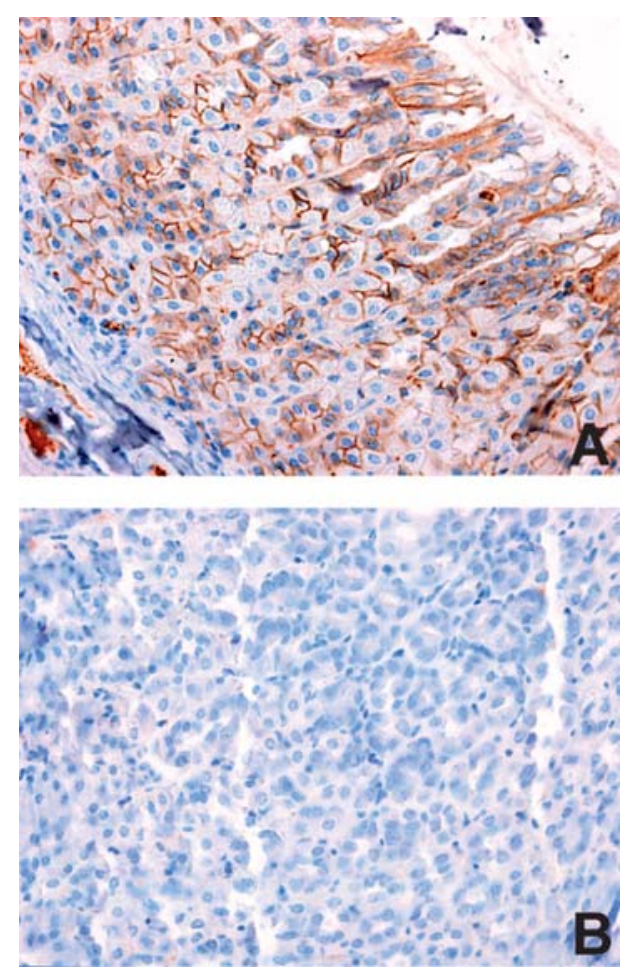

Figure 4. Immunohistochemical detection of mCA IX in the mouse stomach. Tissue sections from the stomach of the wild-type (A) and CA IX-deficient mice (B) were stained with the MAb AM4-3. Strong CA IX staining signal was localized on the basolateral surfaces of gastric epithelial cells in the wildtype stomach, but was completely absent from the CA IX-deficient stomach. Original magnification $\mathrm{x} 400$.

antibodies to corresponding mouse antigen, especially when the antigen is produced in a heterologous system.

The specificity of the antibodies was tested in ELISA against the full-length mouse CA IX antigen ectopically expressed in the mouse NIH3T3 cell line. All monoclonal antibodies bound to extracts from the transfected NIH-mCA IX cells, but not from the control mock-transfected NIH-neo cells. They also recognized GST-mCA IX protein, but not the 
GST only, further supporting their specificity for the mouse CA IX (data not shown).

All the MAbs reacted also in immunoblotting with the mouse CA IX extracted from NIH-mCA IX transfectants as well as with the GST-mCA IX fusion protein (Fig. 1). They could recognize monomeric form of CA IX resolved under reducing conditions (Fig. 1A) as well as the trimer obtained under nonreducing conditions (not shown), and did not produce any background signal with the control CA IXnegative extract from NIH-neo cells. Four MAbs (except the AM34-7 MAb) were also able to immunoprecipitate the biotinylated mouse CA IX protein (Fig. 1B), suggesting that they could recognize both denatured and native mouse CA IX.

All five MAbs can be also used for subcellular localization of mCA IX by immunofluorescence. For this purpose, we tested the MAbs on the methanol-fixed confluent monolayer of the transfected MDCK-mCA IX cells in comparison with the mock-transfected MDCK-neo cells. MDCK cells do not express own CA IX protein but have polarized epithelial phenotype and in that respect are similar to those epithelial cells that naturally express CA IX protein in vivo. Therefore, they are suitable to demonstrate the cell surface localization of the mouse CA IX. Indeed, all the MAbs produced clear plasma membrane signal typical for CA IX in the transfected MDCK cells, whereas the signal was absent from the mock transfected controls (Fig. 2).

Cross-reactivity of anti-mouse CA IX MAbs with human and rat $C A I X$. MAbs specific for the mouse CA IX might potentially recognize related carbonic anhydrase isozymes in other species. This possibility raised a need for evaluation of the cross-reactivity of the new anti-mouse CA IX MAbs with the human and rat antigens. Therefore, we carried out ELISA assay to test the binding of the MAbs to extracts of cells expressing the human and rat CA IX in comparison to monoclonal antibody M75 that is reactive with both human and rat antigens. The extracts were prepared from the human HT-29 colon carcinoma cells that naturally express CA IX in normoxia, NIH3T3 cells transfected with the human CA9 cDNA (NIH-hCA IX), rat sarcoma XC cells and Rat2TKfibroblasts. We found that none of these anti-mouse CA IX MAbs bound to the human CA IX isoform and only one MAb, namely the AM35-1, recognized the rat antigen (data not shown). These results were confirmed in immunoblotting that clearly showed detection of the rat CA IX antigen by AM35-1 antibody (Fig. 3).

Reactivity of the new MAbs in immunohistochemistry. Important feature of the monoclonal antibodies is their capability to specifically react with relevant antigen in sections from paraffin-embedded tissues. Based on the previous studies showing high expression of CA IX in the mouse stomach mucosa, we used the stomach sections to test the MAbs. Stomach sections obtained from the CA IX-deficient mice were used as negative controls. As shown on Fig. 4, MAb AM4-3 was suitable for immunohistochemical detection of the mouse CA IX protein in formalin-fixed, paraffinembedded tissue. The MAb produced typical staining signal localized to membranes of the gastric epithelial cells. The
A

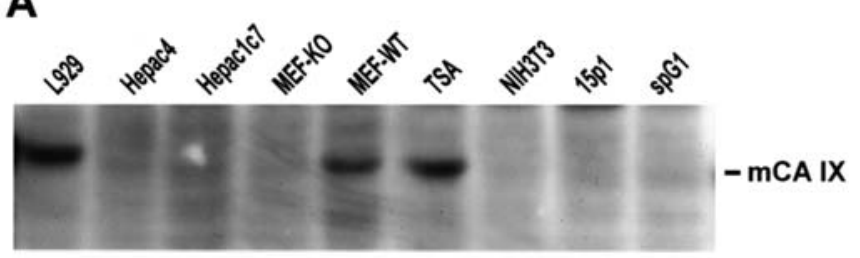

B

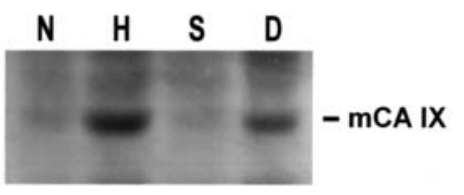

Figure 5. Immunoblot analysis of mCA IX expression/regulation in the mouse cell lines. (A) CA IX expression was induced by hypoxia (48 $\mathrm{h}$ at $2 \% \mathrm{O}_{2}$ ) and detected by MAb AM35-1 in L929 fibroblasts and in TSA cells derived from mammary carcinoma. CA IX was also expressed in hypoxic MEF-wt cells, which contain functional HIF-1 $\alpha$, but not in HIF-1 $\alpha$-deficient cells (MEF-KO). (B) In L929 cells, mCA IX was induced by hypoxia ( H, $48 \mathrm{~h}$ at $2 \% \mathrm{O}_{2}$ ) and in high cell density $\left(\mathrm{D}, 60,000\right.$ cells $/ \mathrm{cm}^{2}$ at plating, $48 \mathrm{~h}$ incubation), but was low or absent in normoxia (N) and under sparse conditions ( $\mathrm{S}, 10,000$ cells $/ \mathrm{cm}^{2}$ at plating) as visualized by AM4-3 MAb.

signal was detectable only in the stomach section from the wild-type animals (Fig. 4A), but not from the CA IX deficient mice (Fig. 4B) supporting its highly specific reactivity against the mouse CA IX protein. Similar staining signal was obtained with the MAbs AM1-4, AM27-4, and AM35-1, whereas the MAb AM34-7 did not work in this immunodetection method (data not shown).

Hypoxia-induced CA IX expression in the mouse cell lines. In contrast to human CA IX that has been thoroughly studied with respect to molecular and functional properties, regulation, expression pattern, as well as various clinical parameters, the mouse ortholog has been so far investigated mostly with respect to its tissue distribution and consequences of its null mutation in developing embryo as well as in adult mouse (16-20). In fact, no consistent data showing expression/ regulation of the mouse CA IX in vitro have been published so far. However, these aspects are critically important for the use of the mouse CA IX as a marker or target in pre-clinical animal models of tumor hypoxia.

With these monoclonal antibodies specifically reactive with the mouse CA IX, we performed immunoblot analysis of endogenous CA IX expression in several mouse cell lines derived from fibroblasts, testicular cells, hepatoma and carcinoma (Fig. 5). CA IX was absent from all tested cell lines grown in subconfluent monolayers under standard normoxic conditions, but could be detected in three cell lines maintained for $48 \mathrm{~h}$ under hypoxia $\left(2 \% \mathrm{O}_{2}\right)$, namely in L929 fibroblasts, wild-type mouse embryonic fibroblasts (MEFwt) and in TSA carcinoma cells (Fig. 5A). The hypoxic induction was confirmed by immunoprecipitation and immuno-fluorescence in TSA and L929 cells (data not shown). In addition, mCA IX expression was upregulated by high cell density (Fig. 5B). These data indicate that the expression of the mouse CA IX is regulated similarly to the human CA IX. 

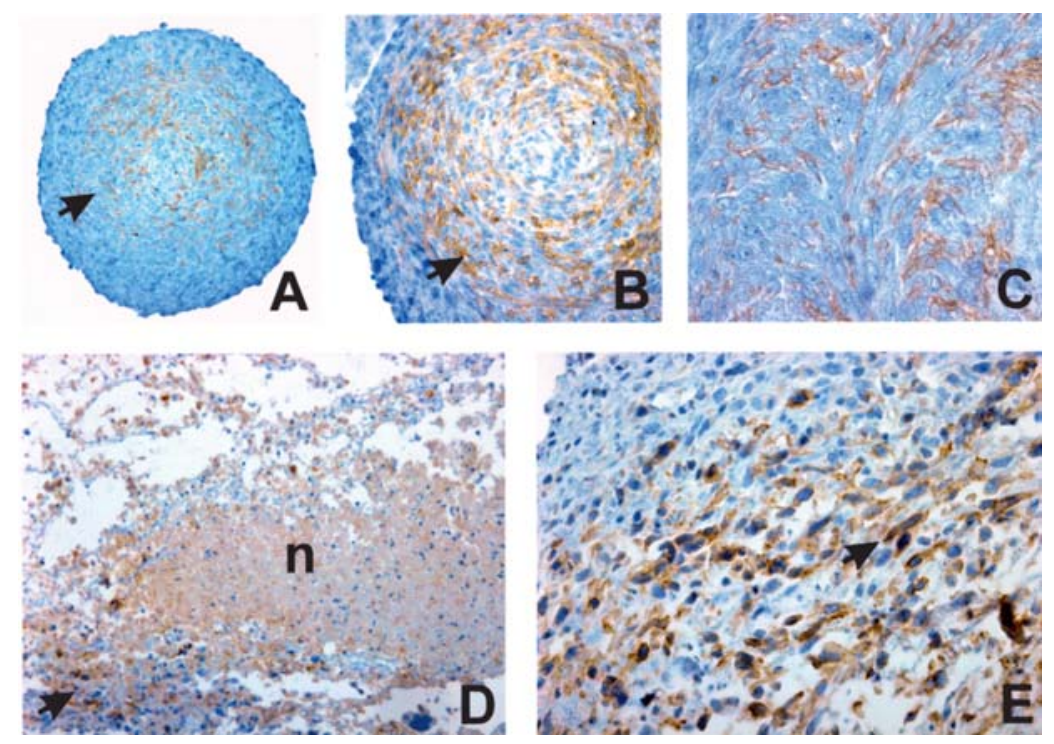

Figure 6. Immunohistochemical analysis of mCA IX expression pattern in multicellular spheroids and in tumor xenografts using AM4-3 MAb. (A and B) Staining of the mCA IX was localized in the intermediate hypoxic layers of multicellular spheroids grown from MEF-wt cells. (C and D) The TSA xenograft exhibited mCA IX staining in the plasma membranes of the perinecrotic cells. The arrows depict the CA IX-positive areas (A, B and D) and a detailed view of the plasma membrane staining signal (E). Original magnification x200 (A and C) x400 (B and D)

CA IX expression in the spheroids and xenografted mouse tumor tissue. Finally, we investigated whether hypoxic regulation of the mouse CA IX observed in cells grown in monolayer was translated to the pattern of CA IX expression in conditions which are more similar to in vivo intratumoral situation, such as three-dimensional multicellular spheroids and tumor xenografts. Out of the three cell lines that showed hypoxia-induced expression of the mouse CA IX, only MEF-wt formed compact spheroids and were suitable for the analysis, whereas TSA spheroids were disintegrated and L929 cells did not grow in 3-D conditions at all. MEF-wt spheroids were fixed in Carnoy's fluid, embedded in paraffin and the sections across the central part were stained with the MAb AM4-3. Fig. 6A and B shows that mCA IX staining signal was absent from well oxygenated cell layers at the surface of spheroid and from the very central necrotic core, but it was clearly visible in the membranes of cells localized in intermediate hypoxic layers. The average distance of the mCA IX-positive cells from the spheroid surface was $66.9 \pm 13.9 \mu \mathrm{m}$ (based on measurements in 12 individual spheroids with average diameter of $474.4 \pm 62.5 \mu \mathrm{m}$ ) and roughly corresponded to expression distance of the human CA IX in hypoxic tumors (21). Also a perinecrotic localization of mCA IX in the mouse tumor xenografts grown from TSA carcinoma cells was fully in accordance with the previous observations in different human tumors (Fig. 6C and D). These data represent a proof of principle for hypoxic regulation of the mouse CA IX and support its meaningful investigation as a hypoxia-related molecular marker/target in the mouse model using the monoclonal antibodies generated and characterized in this study.

\section{Discussion}

Due to its relevance for tumor biology, CA IX has been mostly studied in human tumor cell lines and in tissue specimens as a marker of hypoxia, prognostic indicator and functional component of the hypoxic tumor phenotype (5). The mouse model was first employed a decade after the identification of CA IX in order to investigate its physiological role using a targeted gene disruption approach (16). The same study brought original data on the primary structure of the mouse Car9 cDNA and gene as well as basic information on the expression pattern of Car9 mRNA in embryos and several adult mouse tissues. Most importantly, it clearly demonstrated the role of mCA IX in morphogenesis and homeostasis of the glandular gastric epithelium via the control of cell proliferation and differentiation, since the loss of mCA IX led to hyperplasia of the gastric mucosa with numerous cysts and to disproportion of epithelial cell types (16). These results were extended in the following experiments which revealed that excess dietary salt has no significant effect on the severity of hyperplasia (20).

The study of transgenic animals also resulted in the production of rabbit polyclonal serum raised against mCA IX in order to prove its presence in the wild-type animals and its absence in the deficient mice (16). This polyclonal serum has been subsequently utilized in several studies for more detailed immunoblotting and immunohistochemical assessment of CA IX distribution in the normal mouse tissues. Hilvo et al (17) have confirmed that CA IX is expressed in the stomach mucosa, colon enterocytes and pancreatic acini. Low CA IX protein expression was also observed in the small intestine, testis and brain. Inspection of the female mouse reproductive organs revealed weak CA IX positivity in the endometrial epithelium (18). Moderate to weak CA IX expression has been detected by immunohistochemistry in the developing mouse embryo, namely in the brain, lung, pancreas, liver, kidney and stomach around the embryonic day E12 (19).

These data support the usefulness of the anti-mouse CA IX serum for the immunodetection of CA IX in the mouse 
tissues. However, limited amount of the serum and its polyclonal character are obvious disadvantages which hinder some other applications that require direct labelling of the antibody, domain specificity, compatibility with the mouse immune system for in vivo experiments etc. Based on the overall experiences, these problems can be solved by monoclonal antibodies, which are generally more specific, homogeneous, of limitless availability, can be easily purified and labelled, and recognize a single epitope on the antigen molecule thus allowing for structure-function studies. The monoclonal antibodies generated in CA IX-deficient mice and characterized in this study fulfil those requirements. They can be used as highly specific probes for detection of CA IX in both mouse cell lines and tissues. Specificity of the MAbs is clearly evident from the immunohistochemical staining of the stomach tissue sections. Whereas the stomach specimens from the wild-type mice exhibited typical membrane staining signal in epithelial cells, their CA IX-deficient counterparts were completely negative. Also the immunoblot and immunoprecipitation analyses with the new monoclonal antibodies produced only negligible background. Although we did not use directly labelled MAbs in the present study, our recent experiments have shown that all of them are suitable for this purpose. The new antibodies show differential reactivity in different immunodetection methods suggesting that they do not recognize identical epitopes, however, this remains to be further investigated. Four of the five new antibodies can react with both denatured and native protein and thus can be potentially used for detection of the mouse CA IX in vitro and also for its targeting in vivo. The antibody AM34-7 is most probably directed to a cryptotope localized inside the properly folded native CA IX molecule and can become uncovered upon denaturing with fixatives, extraction buffers or during the blotting procedure.

In addition to demonstration of the immunodetection properties of the new monoclonal antibodies, this study has brought the first direct evidence that the level of the mouse CA IX protein is also regulated by hypoxia and high cell density, similarly to the human CA IX, and that it is expressed in the hypoxic tumor cells and tissues of the mouse origin. Ongoing promoter analyses suggest that the mouse Car9 promoter contains a functional HRE element in the position analogous to that in the human CA9 promoter and that it exhibits similar responsiveness to hypoxia (unpublished data). Nevertheless, the hypoxia-induced expression of the mouse CA IX protein is an important finding favouring the use of the mouse model for the further studies of CA IX role in tumor development and for its preclinical investigations. The monoclonal antibodies described here represent potent tools for accomplishment of these future studies.

\section{Acknowledgements}

We thank Mrs. Kvetoslava Tarabkova for excellent technical assistance. This work was supported by grants from EU (6FP Integrated project EUROXY, LSCH-CT-2003-502932), Slovak Scientific Grant Agency (VEGA 2/6113), Research and Development Support Agency (Contract APVV-51-
024905), Sigrid Juselius Foundation and Academy of Finland.

\section{References}

1. Supuran CT: Carbonic anhydrases: catalytic mechanisms, distribution and physiological roles. In: Carbonic Anhydrase: Its Inhibitors and Activators. Supuran CT, Scozzafava A and Conway J (eds.) CRC Press, Boca Raton, pp1-24, 2004.

2. Parkkila S: An overview of the distribution and function of carbonic anhydrase in mammals. In: The Carbonic Anhydrases: New Horizons. Chegwidden WR, Carter N and Edwards Y (eds.) Birkhauser Verlag, Basel, pp76-93, 2000.

3. Pastorekova S, Parkkila S, Pastorek J and Supuran CT: Carbonic anhydrases: current state of the art, therapeutic applications and future prospects. J Enz Inhib Med Chem 19: 199-229, 2004.

4. Pastorek J, Pastorekova S, Callebaut I, Mornon JP, Zelnik V, Opavsky R, Zatovicova M, Liao S, Portetelle D, Stanbridge E, Zavada J, Burny A and Kettmann R: Cloning and characterization of MN, a human tumor-associated protein with a domain homologous to carbonic anhydrase and putative helix-loop-helix DNA binding segment. Oncogene 9: 2877-2888, 1994.

5. Pastorekova S, Parkkila S and Zavada J: Tumor-associated carbonic anhydrases and their clinical significance. Adv Clin Chem 42: 167-216, 2006.

6. Pastorekova S, Parkkila S, Parkkila AK, Opavsky R, Zelnik V, Saarnio J and Pastorek J: Carbonic anhydrase IX, MN/CA IX: analysis of stomach complementery DNA sequence and expression in human and rat alimentary tracts. Gastroenterology 112: 398-408, 1997

7. Saarnio J, Parkkila S, Parkkila AK, Haukipuro K, Pastorekova S, Pastorek J, Kairaluoma MI and Karttunen TJ: Immunohistochemical study of colorectal tumors for expression of a novel transmembrane carbonic anhydrase, MN/CA IX, with potential value as a marker of cell proliferation. Am J Pathol 153: 279-285, 1998.

8. Wykoff C, Beasley N, Watson P, Turner L, Pastorek J, Sibtain A, Wilson G, Turley H, Talks K, Maxwell PH, Pugh C, Ratcliffe P and Harris AL: Hypoxia-inducible regulation of tumor-associated carbonic anhydrases. Cancer Res 60: 7075-7083, 2000.

9. Kaluz S, Kaluzova M, Chrastina A, Olive PL, Pastorekova S, Pastorek J, Lerman MI and Stanbridge EJ: Lowered oxygen tension induces expression of the hypoxia marker MN/carbonic anhydrase IX in the absence of hypoxia-inducible factor $1 \alpha$ stabilization: a role for phosphatidylinositol 3'-kinase. Cancer Res 62: 4469-4477, 2002.

10. Svastova E, Zilka N, Zatovicova M, Gibadulinova A, Ciampor F, Pastorek J and Pastorekova S: Carbonic anhydrase IX reduces E-cadherin-mediated adhesion of MDCK cells via interaction with B-catenin. Exp Cell Res 290: 332-345, 2003.

11. Svastova E, Hulikova A, Rafajova M, Zatovicova M, Gibadulinova A, Casini A, Cecchi A, Scozzafava A, Supuran CT, Pastorek J and Pastorekova S: Hypoxia activates the capacity of tumor-associated carbonic anhydrase IX to acidify extracellular pH. FEBS Lett 577: 439-495, 2004.

12. Potter CPS and Harris AL: Diagnostic, prognostic and therapeutic implications of carbonic anhydrases in cancer. Br J Cancer 89: 2-7, 2003.

13. Robertson N, Potter C and Harris AL: Role of carbonic anhydrase IX in human tumor cell growth, survival, and invasion. Cancer Res 64: 6160-6165, 2004.

14. Pastorekova S, Zavadova Z, Kostal M, Babusikova O and Zavada J: A novel quasi-viral agent, MaTu, is a two-component system. Virology 187: 620-626, 1992 .

15. Zatovicova M, Tarabkova K, Svastova E, Gibadulinova A, Mucha V, Jakubickova L, Biesova Z, Rafajova M, Gut MO, Parkkila S, Parkkila AK, Waheed A, Sly WS, Horak I, Pastorek J and Pastorekova S: Monoclonal antibodies generated in CA IXdeficient mice recognize different domains of tumor-associated hypoxia-induced carbonic anhydrase IX. J Immunol Methods 282: 117-134, 2003 .

16. Gut MO, Parkkila S, Vernerova Z, Rohde E, Zavada J, Hocker M, Pastorek J, Karttunen T, Gibadulinova A, Zavadova Z, Knobeloch KP, Wiedenmann B, Svoboda J, Horak I and Pastorekova S: Gastric hyperplasia in mice with targeted disruption of the carbonic anhydrase gene Car9. Gastroenterology 123: 1889-1903, 2002. 
17. Hilvo M, Rafajová M, Pastoreková S, Pastorek J and Parkkila S: Expression of carbonic anhydrase IX in mouse tissues. J Histochem Cytochem 52: 1313-1322, 2004.

18. Hynninen P, Hamalainen JM, Pastorekova S, Pastorek J, Waheed A, Sly WS, Tomas E, Kirkinen P and Parkkila S: Transmembrane carbonic anhydrase isozymes IX and XII in the female mouse reproductive organs. Reprod Biol Endocrinol 2: 73-, 2004.

19. Kallio H, Pastorekova S, Pastorek J, Waheed A, Sly WS, Mannisto S, Heikinheimo M and Parkkila S: Expression of carbonic anhydrases IX and XII during mouse embryonic development. BMC Dev Biol 6: 22, 2006.
20. Leppilampi M, Karttunen TJ, Kivela J, Gut MO, Pastorekova S, Pastorek J and Parkkila S: Gastric pit cell hyperplasia and glandular atrophy in carbonic anhydrase IX knockout mice: studies on two strains C57/BL6 and BALB/C. Transgenic Res 14: 655-663, 2005.

21. Beasley NJP, Wykoff CC, Watson PH, Leek R, Turley H, Gatter K, Pastorek J, Cox GJ, Ratcliffe P and Harris AL: Carbonic anhydrase IX, an endogenous hypoxia marker, expression in head and neck squamous cell carcinoma and its relationship to hypoxia, necrosis, and microvessel density. Cancer Res 61: 5262-5267, 2001. 